Swarthmore College

Works

3-10-2012

\title{
Three-Dimensional Phase Step Profilometry With A Multicore Optical Fiber
}

Ari Jason Novack , '12

David Joseph D'Annunzio , '12

Ekin Dogus Çubuk , '10

N. Inci

Lynne Molter , '79

Swarthmore College, Imolter1@swarthmore.edu

Follow this and additional works at: https://works.swarthmore.edu/fac-engineering

Part of the Engineering Commons

Let us know how access to these works benefits you

\section{Recommended Citation}

Ari Jason Novack , '12; David Joseph D'Annunzio , '12; Ekin Dogus Çubuk , '10; N. Inci; and Lynne Molter , '79. (2012). "Three-Dimensional Phase Step Profilometry With A Multicore Optical Fiber". Applied Optics. Volume 51, Issue 8. 1045-1048. DOI: 10.1364/A0.51.001045

https://works.swarthmore.edu/fac-engineering/29

This work is brought to you for free by Swarthmore College Libraries' Works. It has been accepted for inclusion in Engineering Faculty Works by an authorized administrator of Works. For more information, please contact myworks@swarthmore.edu. 


\title{
Three-dimensional phase step profilometry with a multicore optical fiber
}

\author{
Ari Novack, ${ }^{1, *}$ David D’Annunzio, ${ }^{1}$ Ekin Doğuş Çubuk, ${ }^{1,2}$ Naci Inci, ${ }^{3}$ and Lynne Molter ${ }^{1}$ \\ 'Department of Engineering, Swarthmore College, 500 College Avenue, Swarthmore, Pennsylvania 19081, USA \\ ${ }^{2}$ School of Engineering and Applied Sciences, Harvard University, 1350 Massachusetts Avenue, \\ Cambridge, Massachusetts 02138, USA \\ ${ }^{3}$ Physics Department, Boğaziçi University Bebek 34342, Istanbul, Turkey \\ ${ }^{*}$ Corresponding author: arinovack@gmail.com
}

Received 23 September 2011; revised 10 November 2011; accepted 11 November 2011; posted 15 November 2011 (Doc. ID 154642); published 5 March 2012

\begin{abstract}
This paper demonstrates the feasibility of using phase stepping and a multicore optical fiber to calculate an object's depth profile. An interference pattern is projected by an optical fiber onto the object. The distorted interference pattern containing the object information is captured by a CCD camera and processed using a phase step interferometry method. The phase step method is less computationally intensive compared to two-dimensional Fourier transform profilometry and provides more accuracy when measuring objects of high frequency spatial variations. (C) 2012 Optical Society of America

OCIS codes: $\quad 060.2310,120.2650,120.2830,120.5050$.
\end{abstract}

\section{Introduction}

Three-dimensional profilometry is the measurement of an object's depth using noninvasive methods and is useful in many fields, including artwork inspection, object recognition, and roughness analysis [1-3] . One method of profiling an object is to project an interference pattern onto an object and then analyze the pattern to determine the depth map of the object.

A standard practice to generate an interference pattern is to use two single-core fibers [1, $\underline{1}]$. A fiber coupler is typically used to achieve coherence between the two fibers. The outputs of the fibers act as coherent point sources. A multicore fiber can also be used to generate an interference pattern using the individual cores as the coherent point sources. Using a single fiber optic cable with multiple cores adds tremendous stability to what would normally be a fragile system. By combining the fiber cores into one cladding, the need for a fiber coupler is eliminated.

$1559-128 \mathrm{X} / 12 / 081045-04 \$ 15.00 / 0$

(C) 2012 Optical Society of America
Vibration, temperature, and other external factors have less effect making the system more robust.

This paper is an extension of the multicore Fourier transform work of Bulut and Inci [5]. With similar equipment and setup, but using the three-step phase-shift method instead of the Fourier transform method, the computational intensity required for image processing can be reduced, and the accuracy of reproducing higher spatial frequency data can be increased. The reduced computational intensity of the phase-shift method could potentially allow for image processing to be done by a cheap microprocessor. The Fourier transform method is limited in its ability to show higher spatial frequencies due to the necessity of applying a low-pass filter to the frequency data.

Light was coupled into two cores of a four-core optical fiber and projected onto an object. Only two cores of the four-core fiber were used because employing a two-point instead of a four-point interference pattern increased the phase-shifting accuracy and decreased the time needed for the phase shifting. The interference pattern projected by this source was deformed by the surface of the object. Three images were taken with the phase shifted by $\pi / 2$ 
between each pattern. These images were then used to calculate the object's depth profile.

\section{Theory}

Since two adjacent cores of the fiber were used, the fringe analysis can be done for a two-point source as opposed to a four-point source. Acting as two coherent sources, the laser light creates an interference pattern described as []ㅡ,

$$
\begin{gathered}
I(x, y)=2 I_{0}(1+\cos (A(x \cos \theta-z(x, y) \sin \theta))), \\
A=\frac{2 \pi \delta}{\lambda f},
\end{gathered}
$$

where $\delta$ is the separation between fiber ends, $\lambda$ is the wavelength of light, and $f$ is the distance from the end of the fiber to the object. $I_{0}$ is the light intensity of a single core, and $z(x, y)$ is the depth map of the object. As shown in Appendix A, the phase at the object's surface $(\phi(x, y))$ is given by

$$
\phi(x, y)=\tan ^{-1}\left(\frac{I_{2}-I_{3}}{I_{2}-I_{1}}\right)=A(x \cos \theta-z(x, y) \sin \theta) .
$$

Plugging three different images with phase shifts of $\pi / 2$ into (3) can be used to obtain the depth profile, $z(x, y)$.

$$
z(x, y)=\frac{A x \cos \theta-\phi(x, y)}{A \sin \theta} .
$$

The arctan function returns the phase value in wrapped form that is in the range $(-\pi<\phi \leq \pi)$. An unwrapping algorithm must be used to find the true phase [6-8].

\section{Experiment}

An infrared beam with a wavelength of $798 \mathrm{~nm}$ is emitted from a titanium-sapphire laser, pumped by a $5 \mathrm{~W}, 532 \mathrm{~nm}$ beam. The beam is directed into a $110 \mathrm{~cm}$ four-core fiber. The light is focused into two adjacent cores so that the fiber behaves equivalently to a two-core fiber. Each core is $10.6 \mu \mathrm{m}$ in diameter with a core separation of $40.6 \mu \mathrm{m}$. After the light travels the length of the fiber, the resulting interference pattern is projected onto the object, $49 \mathrm{~cm}$ from the output end of the cable (see Fig. 1). The pattern has a projection angle of approximately $21^{\circ}$ from perpendicular. This angle is minimized to decrease the areas of shadow that contain no phase information. The resulting image is captured by the CCD camera, which has a line of focus perpendicular to the object plane.

A phase shift with an error of 0.01 radians or less must be obtained between the images. A micrometer mount is used to bend a constrained section of the fiber. The light travels further on the outside core

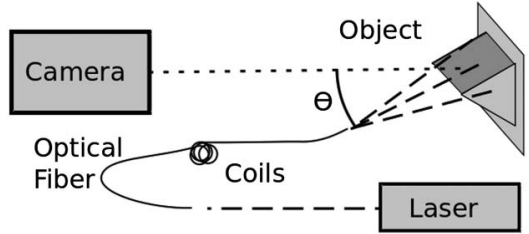

Fig. 1. Experimental setup.

of the bend than on the inside core, resulting in a phase shift $[9,10]$.

After acquiring a photo at each of the three consecutive phase steps, the images are uploaded to the computer and processed using MATLAB software. Once the wrapped phase map is obtained from the images, the phase is unwrapped using the block-least squares algorithm [8]. This algorithm divides the domain into blocks, unwraps each block individually, and then merges the blocks by minimizing discontinuities. Block-least squares was chosen specifically for its ability to quickly unwrap the phase while avoiding discontinuities due to noise.

\section{Results}

A number of objects were profiled using this method, of which two are shown in Figs. $\underline{4}$ and 5 . The first object is a triangular prism, $24 \mathrm{~mm}$ in width and $17.2 \mathrm{~mm}$ in height. Figure $\underline{2}$ shows the comparison of a cross section from the triangular prism with the result using the phase step method. The rootmean-square error is $0.7 \mathrm{~mm}$.

It is important to note that the reported technique is limited when applied to dark objects or objects of high reflectivity. Dark objects had lower signal-tonoise ratios and were harder to unwrap. On the other hand, some metal and plastic objects were too reflective, causing the interference pattern to saturate the CCD camera. This effect is mainly due to absorption or scattering of light and is directly related to the dynamic range of the camera used, but not related to the method itself. Using a camera with a higher dynamic range would allow us to model a wider variety of objects.

The largest source of noise was speckle noise, which is caused by the interference of the light with itself due to small path deviations. Smoother objects decreased the speckle, increased the signal-to-noise ratio, and produced higher quality depth maps. White plastic and standard white printer paper

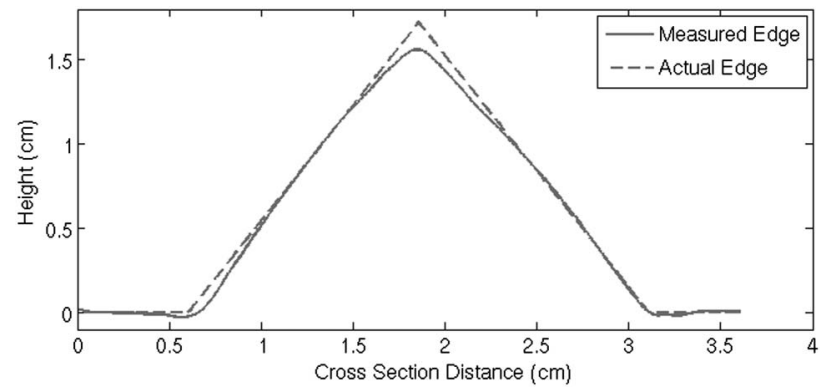

Fig. 2. Profile of a triangle. 


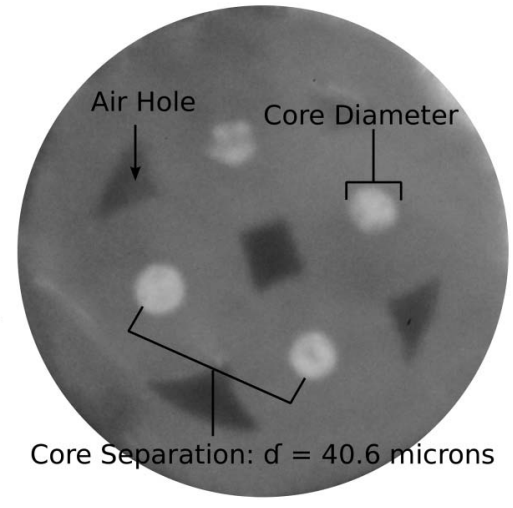

Fig. 3. Profile of a cylinder.

produced good surfaces with a high reflectivity and satisfactory smoothness.

\section{Discussion}

The use of a multicore optical fiber provides sizable advantages over a two-fiber system. With a multicore fiber, the setup is simpler, cheaper, and easier to maintain. There is no need for a fiber coupler to produce the two coherent sources for the interference pattern. The use of a single fiber eliminates the need for adjusting the path difference between two fibers and calibrating a phase-stepping transducer.

A four-core fiber as shown in Fig. $\underline{3}$ is employed in our experiments. All cores are $10.6 \mu \mathrm{m}$ in diameter and are separated by a distance of $40.6 \mu \mathrm{m}$. The separation between cores is large enough that coupling from one core to another is negligible. Each core is designed to guide a wavelength of approximately $1.3 \mu \mathrm{m}$. The fiber is coiled a number of times before phase stepping to completely filter out the higher modes so that only a single mode at $798 \mathrm{~nm}$ remains. Since each fiber core guides a single mode, the bending during the phase-stepping process only affects the phase of the signals in each core, and not their modal pattern.

Since only two cores of the four-core fiber were needed, the problem of coupling into just two cores would have been solved by employing a two-core fiber, but none were available. Additionally, only four to five fringes were projected by the fiber. A larger

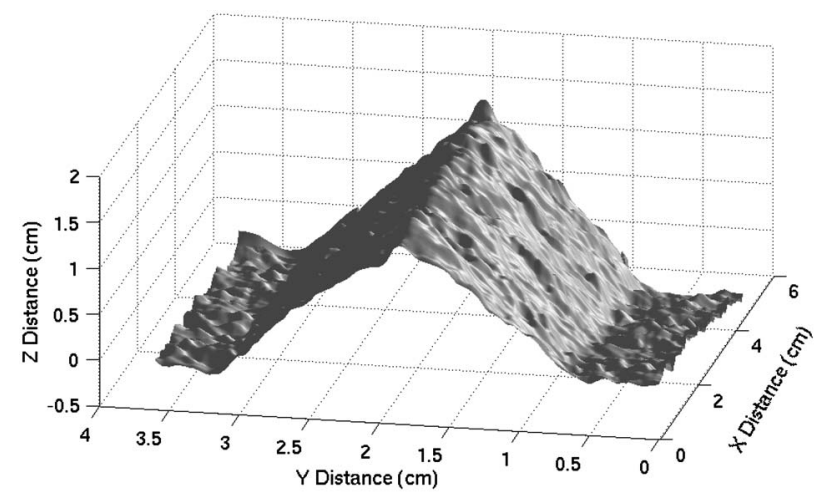

Fig. 4. Result comparison for triangular object.

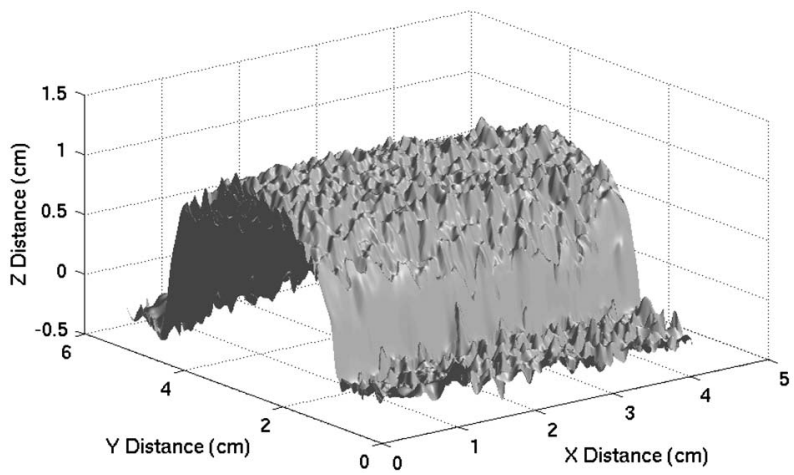

Fig. 5. Four-core fiber cross section.

number of fringes compacted into the same space would increase the accuracy of the system. The core separation could be increased to allow for more fringes using the same wavelength.

As noted above, the largest single source of error was speckle. The most successful technique used to minimize speckling was to vibrate the object and background to average out the speckles. Increasing the amount of vibration decreased the noise due to speckle, but also decreased accuracy due to the image blurring.

The drawback of the phase step method is the need to take multiple pictures and the difficulty in controlling the phase stepping. The phase must be adjusted extremely accurately between pictures. When two single-core fibers are used, the phase can be shifted by extending one path relative to the other. This is often done with a mirror mounted on a precisely calibrated piezoelectric transducer [11]. As both light paths in the setup are wrapped in a single cladding, one path cannot be isolated and lengthened. Instead, a micrometer was used to bend the fiber and move the fringes into the correct position. This was an unpredictable method for phase shifting, as the amount of bending was not linearly related to the phase shift. An automated fringe tracking system was employed to direct the manually phase-shifted fringe pattern. Because of the relative nature of the phase-stepping process, the fringe tracking system was able to compensate for phase shifts due to vibration and temperature fluctuations.

Currently, the phase-stepping procedure is limiting the speed of the method. It takes 20-30 seconds to take the three pictures needed to calculate the depth profile. This time can be reduced by using a fiber in which a higher percentage of light is coupled. This would allow for a higher frame rate from the camera, essential for accelerating the capture of all three photos. A servo motor could also be introduced to allow for high speed shifting with a greater degree of accuracy than the manual shifting method. If all of these methods were employed, it is reasonable to estimate a capture time around $1 \mathrm{~s}$, allowing for a depth profile stream of about the same speed.

Object discontinuities also pose a difficulty for this method. Since phase wrapping is done by correcting 
phase discontinuities, any physical discontinuity larger than half of a fringe $(\pi)$ has a chance of causing unwrapping errors.

\section{Conclusion}

The feasibility of using a multicore fiber optic cable in obtaining a depth profile with the phase-shift method has been demonstrated. The system is compact and reliable. Sensitivity can be increased with an increase in the number fringes, a reduction in speckling, and the use of a two-core fiber. The method of bending the fiber to obtain the necessary phase shifts has been shown to be practical. The method could be improved by the use of a modified fiber, along with a servo motor, which would enable a streaming three-dimensional image with a frame rate around one Hertz.

\section{Appendix A: Three-Step Phase-Shift Derivation}

The output from the optical fiber is a two-point source with an intensity pattern:

$$
I_{1}=2 I_{0}(1+\cos (A x \cos \theta-A z \sin \theta)) .
$$

Each subsequent image is taken with a $\pi / 2$ phase shift such that the other two image intensities are given by

$$
\begin{aligned}
I_{2} & =2 I_{0}(1+\cos (A x \cos \theta-A z \sin \theta+\pi / 2)) \\
& =2 I_{0}(1-\sin (A x \cos \theta-A z \sin \theta)), \\
I_{3} & =2 I_{0}(1+\cos (A x \cos \theta-A z \sin \theta+\pi)) \\
& =2 I_{0}(1-\cos (A x \cos \theta-A z \sin \theta)),
\end{aligned}
$$

$\frac{I_{2}-I_{3}}{I_{2}-I_{1}}=\frac{\cos (A x \cos \theta-A z \sin \theta)-\sin (A x \cos \theta-A z \sin \theta)}{\cos (A x \cos \theta-A z \sin \theta)+\sin (A x \cos \theta-A z \sin \theta)}$.

Using $\quad \sin (x)+\cos (x)=\sqrt{2} \cos (x-\pi / 4) \quad$ and $\sin (x)-\cos (x)=\sqrt{2} \sin (x-\pi / 4)$, we rewrite the intensities as

$$
\begin{aligned}
\frac{I_{2}-I_{3}}{I_{2}-I_{1}} & =\frac{\sin (A x \cos \theta-A z \sin \theta-\pi / 4)}{\cos (A x \cos \theta-A z \sin \theta-\pi / 4)} \\
& =\tan (A x \cos \theta-A z \sin \theta-\pi / 4)
\end{aligned}
$$

The extraneous $\pi / 4$ is thrown out since the starting phase does not matter. Rearranging this equation, $z$ can be isolated,

$$
z=\frac{A x \cos \theta-\phi}{A \sin (\theta)}
$$

where the phase $(\phi)$ is given by

$$
\phi=\tan ^{-1}\left(\frac{I_{2}-I_{3}}{I_{2}-I_{1}}\right)
$$

We would like to thank the Halpern Family Fund for financial support.

\section{References}

1. G. Schirripa Spagnolo, G. Guattari, C. Sapia, D. Ambrosini, D. Paoletti, and G. Accardo, "Three-dimensional optical profilometry for artwork inspection,” J. Opt. Soc. Am. A 2, 353361 (2000).

2. J. J. Esteve-Taboada, D. Mas, and J. Garca, "Threedimensional object recognition by Fourier transform profilometry," Appl. Opt. 38, 4760-4765 (1999).

3. L. De Chiffre, S. Christiansena, and S. Skadea, "Advantages and industrial applications of three-dimensional surface roughness analysis," CIRP Ann. Manufacturing Technol. 43 473-478 (1994).

4. T. L. Pennington, H. Xiao, R. May, and A. Wang, "Miniaturized 3-D surface profilometer using a fiber optic coupler," Opt. Laser Technol. 33, 313-320 (2001).

5. K. Bulut and N. Inci, "Three-dimensional optical profilometry using a four-core optical fiber," Opt. Laser Technol. 37, 463469 (2005)

6. D. Ghiglia and M. Pritt, Two-Dimensional Phase Unwrapping, Theory, Algorithms, and Software (Wiley, 1998).

7. J. Strand and T. Taxt, "Performance evaluation of twodimensional phase unwrapping algorithms," Appl. Opt. 38, 4333-4344 (1999).

8. J. Strand, T. Taxt, and A. Jain, "Two-dimensional phase unwrapping using a block least-squares method," IEEE Trans. Image Process. 8, 375-386 (1999).

9. M. J. Gander, D. Macrae, E. A. C. Galliot, R. McBride, J. D. C. Jones, P. M. Blanchard, J. G. Burnett, A. H. Greenaway, and M. N. Inci, "Two-axis bend measurement using multicore optical fibre," Opt. Commun. 182, 115-121 (2000).

10. S. Zhao, X. Wang, and L. Yuan, "Four-core fiber-based bending sensor," Optoelectron. 1, 231-236 (2008).

11. C. Ai and J. Wyant, "Effect of piezoelectric transducer nonlinearity on phase shift interferometry," Appl. Opt. 26, 1112-1116 (1987). 